\title{
Analysis of Lipid-Associated Gene Expression in Mouse Endotoxin Model
}

Charles Price ${ }^{1}$, Takashi Hato ${ }^{2}$

${ }^{1}$ Indiana University School of Medicine, ${ }^{2}$ Indiana University School of Medicine, Department of Medicine, Division of Nephrology

\section{Background/Objective:}

Sepsis-induced kidney injury is a major clinical problem and is an independent risk factor for mortality. We and others have reported that renal tissue metabolism is profoundly altered in the septic milieu. However, whether such metabolic shift is an adaptive response or pathologic process remains unclear. For instance, decreased lipid metabolism in the renal peroxisomes could limit the genesis of deleterious reactive oxygens species whereas the lack of lipid usage in peroxisomes could contribute to energy depletion. Accordingly, here we examined gene expression changes involved in lipid metabolism using mouse models of endotoxemia. Specifically, we compared gene expression changes between injurious high-dose endotoxemia and protective low-dose endotoxin preconditioning models with the goal of identifying favorable reprogramming in lipid metabolism.

\section{Methods:}

To induce protective preconditioning, mice were subjected to low-dose endotoxin followed 24 hours later by high-dose endotoxin. Non-preconditioned mice were subjected to a single highdose endotoxin. Renal tissues were processed and single-cell RNA-sequencing was performed. Tissue metabolomic data was also obtained.

\section{Results:}

We found that renal lipid metabolism is deranged in the non-preconditioned endotoxemia model. Plin2, a marker for lipid accumulation, was significantly upregulated in the proximal tubules of non-preconditioned animals. In contrast, Plin2 expression was preserved in the preconditioned animals, suggesting that altered Plin2 expression is a maladaptive response. Similarly, Pdzk1 and Lrp2, genes involved in HDL/LDL receptor expression, were concurrently downregulated in the injurious model, whereas their expression levels were preserved in the protective model. The stable expression of these two genes may be important for maintaining metabolic capacity and anti-inflammatory effects observed in the preconditioned state. These findings were further supported by our tissue metabolomics analysis.

\section{Conclusion/Impact:}

Our data indicate that aberrant lipid metabolism is a prominent feature of endotoxin-induced kidney injury. The use of protective preconditioning served as a platform to identify several candidate genes that could be studied further for the development of biomarkers and precise intervention. 